\title{
Rapid Orbital Decay in Detached Binaries: Evidence for Circumbinary Disks
}

\author{
Wen-Cong Chen ${ }^{1,2,3}$ and Philipp Podsiadlowski ${ }^{2,3}$ \\ ${ }^{1}$ School of Physics and Electrical Information, Shangqiu Normal University, Shangqiu 476000, China; chenwc@pku.edu.cn \\ ${ }^{2}$ Department of Physics, University of Oxford, Oxford OX1 3RH, UK \\ 3 Argelander-Insitut für Astronomie, Universität Bonn, Auf dem Hügel 71, D-53121 Bonn, Germany \\ Received 2016 October 14; revised 2017 February 20; accepted 2017 February 21; published 2017 March 7
}

\begin{abstract}
Some short-period, detached binary systems have recently been reported as experiencing very rapid orbital decay, much faster than is expected from the angular-momentum loss caused by gravitational radiation alone. As these systems contain fully convective stars, magnetic braking is not believed to be operative, making the large orbitalperiod derivative puzzling. Here, we explore whether a resonant interaction between the binary and a surrounding circumbinary (CB) disk could account for the observed orbital decay. Our calculations indicate that the observed orbital-period derivatives in seven detached binaries can be produced by the resonant interaction between the binary and a CB disk if the latter has a mass in the range of $10^{-4}-10^{-2} M_{\odot}$, which is of the same order as the inferred disk mass $\left(\sim 2.4 \times 10^{-4} M_{\odot}\right)$ in the post-common-envelope binary NN Ser.
\end{abstract}

Key words: binaries: close - stars: evolution - stars: individual (NN Ser) - stars: low-mass - white dwarfs

\section{Introduction}

Interacting binaries are generally systems in which one or both components overflow their Roche lobe(s); they are ideal probes for testing stellar and binary evolutionary theory. During the evolution of interacting binaries, orbital angular-momentum loss mechanisms play a vital role in driving mass transfer and the orbital evolution. The most important of these are due to gravitational radiation, mass-loss, and magnetic braking. However, it is often difficult in interacting binaries to untangle the effects of angular-momentumloss from the effects of mass transfer.

In contrast, in detached binaries there is usually no significant mass transfer; this makes it possible-in principle - to constrain any systemic angular-momentum-loss directly from an orbital-period change. Recently, some detached binaries have been found to experience very rapid orbital decay. The detached eclipsing binary SDSS J143547.87 +373338.5 (hereafter J1435), with an orbital period of 0.126 days, has a white dwarf primary (Eisenstein et al. 2006) and a companion star of spectral type M4-M6 (Steinfadt et al. 2008), which is most likely fully convective. Qian et al. (2016) reported that the orbital period of this system decreases continuously at a rate $\dot{P}=-8.04 \times 10^{-11} \mathrm{~s} \mathrm{~s}^{-1}$. Generally, orbital-period changes in binary systems may be caused by three mechanisms: (1) genuine orbital angularmomentum-loss, (2) the so-called Applegate mechanism (Applegate 1992), and (3) the existence of a third body in the system. Gravitational radiation alone only produces a period derivative of $\dot{P}_{\mathrm{gr}}=-8.1 \times 10^{-14} \mathrm{~s} \mathrm{~s}^{-1}$, which is three orders of magnitude smaller than the observed value. Meanwhile, in the standard theory a fully convective star does not produce magnetic braking (Verbunt \& Zwaan 1981; Rappaport et al. 1983; Spruit \& Ritter 1983), a key ingredient for explaining the period gap of cataclysmic variables.

In Table 1, we summarize the relevant parameters for seven detached binary systems with an observed orbital-period derivative. For a detached binary, the orbital-period derivative induced by gravitational radiation is given by

$$
\dot{P}_{\mathrm{gr}}=-\frac{96 G^{3}}{5 c^{5}} \frac{M_{1} M_{2}\left(M_{1}+M_{2}\right)}{a^{4}} P,
$$

where $G$ is the gravitational constant, $c$ is the speed of light in vacuo, $a$ is the orbital separation, $P$ is the orbital period, and $M_{1}$ and $M_{2}$ are the masses of the two components, respectively. As the table shows, the orbital-period derivatives that can be caused by gravitational radiation alone are 1-5 orders of magnitude smaller than the values observed.

If one assume that the secondary $\left(M_{2}\right)$ experiences magnetic braking, the standard model for magnetic braking given by Rappaport et al. (1983) predicts an orbital-period derivative

$$
\begin{aligned}
\dot{P}_{\mathrm{mb}}= & -1.4 \times 10^{-12}\left(\frac{M_{\odot}}{M_{1}}\right)\left(\frac{M_{1}+M_{2}}{M_{\odot}}\right)^{1 / 3} \\
& \times\left(\frac{R_{2}}{R_{\odot}}\right)^{\gamma}\left(\frac{d}{P}\right)^{7 / 3} \mathrm{~s} \mathrm{~s}^{-1} \cdot
\end{aligned}
$$

Even if magnetic braking were not to cease completely for stars without radiative cores (King et al. 2002; Andronov et al. 2003; Pretorius \& Knigge 2008; Qian et al. 2015), this mechanism (with the standard value of $\gamma=4$ ) could not be responsible for the rapid orbital decay in these seven detached binaries (see Table 1).

If the observed period decrease is just a short-term phenomenon (rather than a secular decrease), it could possibly arise from the Applegate mechanism (Applegate 1992). It is generally thought that the stellar magnetic field results from magnetic dynamo action. Here, the interplay between differential rotation and cyclonic convection induces a magnetic activity cycle as follows: the shear due to the differential rotation drives a poloidal field to form a toroidal subsurface field; due to the convection, the toroidal field moves to the star's surface and twists it; finally, the Coriolis force converts this twist into a poloidal field, thus finishing the cycle (Applegate 1992). In this picture, the magnetic activity induces angular-momentum transfer between different convective zones of the cool star, changing the rotational velocity of the star, and hence, due to the tidal locking, the orbital period of the binary. However, the energy necessary to make this mechanism operate cannot be provided by the total radiation 
Table 1

Key Parameters for Seven Detached Binary Systems with Observed Orbital-period Derivatives

\begin{tabular}{|c|c|c|c|c|c|c|c|c|c|c|}
\hline Sources & Primary Types & $\begin{array}{c}M_{1} \\
\left(M_{\odot}\right)\end{array}$ & $\begin{array}{c}M_{2} \\
\left(M_{\odot}\right)\end{array}$ & $\begin{array}{c}R_{2} \\
\left(R_{\odot}\right)\end{array}$ & $\begin{array}{c}P \\
\text { (days) }\end{array}$ & $\begin{array}{c}a \\
\left(R_{\odot}\right)\end{array}$ & $\begin{array}{c}\dot{P} \\
\left(10^{-11} \mathrm{~s} \mathrm{~s}^{-1}\right)\end{array}$ & $\begin{array}{c}\dot{P}_{\mathrm{gr}} \\
\left(10^{-13} \mathrm{~s} \mathrm{~s}^{-1}\right)\end{array}$ & $\begin{array}{c}\dot{P}_{\mathrm{mb}}^{\mathrm{a}} \\
\left(10^{-13} \mathrm{~s} \mathrm{~s}^{-1}\right)\end{array}$ & References \\
\hline $\mathrm{J} 1435$ & white dwarf & 0.5 & 0.21 & 0.23 & 0.126 & 0.94 & -8.04 & -0.81 & -8.8 & $1,2,3$ \\
\hline V2051 Oph & white dwarf & 0.78 & 0.15 & 0.15 & 0.0625 & 0.65 & -0.162 & -2.6 & -5.7 & 4,5 \\
\hline NN Ser & white dwarf & 0.535 & 0.111 & 0.18 & 0.13 & 0.93 & -0.60 & -0.45 & -2.8 & $6,7,8$ \\
\hline HU Aqr & white dwarf & 0.88 & 0.20 & 0.22 & 0.087 & 0.85 & -0.56 & -2.2 & -11.4 & 9,10 \\
\hline NY Vir & subdwarf B & 0.46 & 0.14 & 0.18 & 0.101 & 0.77 & -0.92 & -0.76 & -5.7 & 11,12 \\
\hline HW Vir & subdwarf B & 0.48 & 0.14 & 0.18 & 0.117 & 0.86 & -2.12 & -0.61 & -3.9 & 13,14 \\
\hline WY Cancri & G5 & 0.81 & 0.31 & 0.58 & 0.829 & 3.85 & -33.0 & -0.07 & -3.1 & 15,16 \\
\hline
\end{tabular}

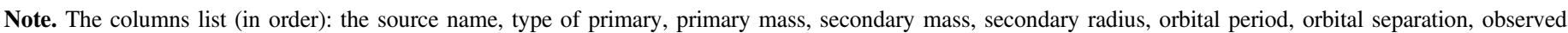
orbital-period derivative, orbital-period derivative induced by gravitational radiation, orbital-period derivative induced by magnetic braking, and references.

${ }^{a}$ We only consider the magnetic braking contribution of secondary stars.

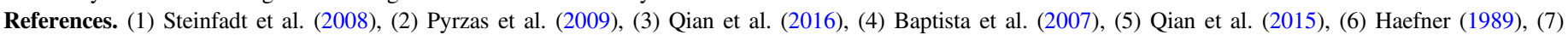

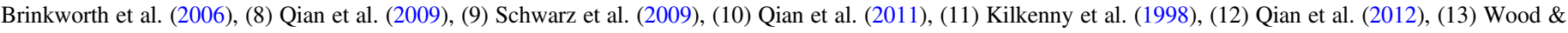
Saffer (1999), (14) Qian et al. (2008), (15) Zeilik et al. (1990), (16) Heckert et al. (1998).

energy in the case of NN Ser (Brinkworth et al. 2006) and V2051 Oph (Qian et al. 2015). Therefore, it is still a puzzle why these detached binaries experience such rapid orbital shrinking. The solution of this problem may offer some more general, valuable clues for the theory of single and binary stars.

Assuming that the orbital-period changes in these seven detached binaries originate from genuine angular-momentum loss from the binary, in this Letter we attempt to diagnose whether a circumbinary (CB) disk around these systems could provide the solution by causing the transfer of orbital angular momentum from the binary to the disk. In Section 2, we describe the CB disk model and compare the predicted orbitalperiod derivatives with the observed values. In Section 3, we summarize the results with brief conclusions and a discussion.

\section{CB Disk Model}

The existence of CB disks has previously been deduced from the analysis of the blackbody spectrum in the black hole X-ray binaries A0620-00 and XTE J1118+480. Muno \& Mauerhan (2006) found that the inferred emitting surface areas of the observed excess mid-infrared emission are approximately two times larger than the binary orbit. Subsequent observations with the Wide-field Infrared Survey Explorer have indicated that these two sources may hide CB disks (Wang \& Wang 2014). Recently, ALMA detection of flux at $1.3 \mathrm{~mm}$ for the post-common-envelope binary $\mathrm{NN}$ Ser has been proposed to likely originate from the thermal emission from a dust disk with a mass of $2.4 \pm 0.6 \times 10^{-6} M_{\odot}$ (Hardy et al. 2016). These observations provide strong evidence that $\mathrm{CB}$ disks may exist around some detached binaries. So far, the process by which such $\mathrm{CB}$ disks form has not yet been fully understood. CB disks may be produced by some commonenvelope material that has not been ejected from the system for a binary like NN Ser. Alternatively, the origin of a CB disk may be closely related to the mass outflow processes during mass transfer. For example, it has been argued in the case of postulated CB disks around millisecond pulsar binaries that, during hydrogen-shell flashes in the helium secondary, the transferred material exceeding the Eddington accretion rate may be expelled through $\mathrm{L} 2$, causing the formation of a $\mathrm{CB}$ disk (Antoniadis 2014).
Because of the relatively large orbital angular momentum, mass-loss during mass transfer may form a disk surrounding the binary system rather than leave it (van den Heuvel \& de Loore 1973; van den Heuvel 1994). Assuming a small fraction of the transferred mass feeds a CB disk, Spruit \& Taam (2001) and Taam \& Spruit (2001) found that the torque provided by the $\mathrm{CB}$ disk could produce a range of variation of mass transfer rates that is compatible with the range of mass transfer rates seen in cataclysmic variables. Other relevant studies show that the existence of a CB disk can provide an efficient angularmomentum loss mechanism, which enhances the mass transfer rate and causes the secular shrinking of the binary orbit (Chen \& Li 2006, 2007, 2015; Chen et al. 2006).

We now assume that the seven detached binaries with rapid orbital decay are surrounded by CB disks. Recent MHD simulations show that, for the mass ratios applicable to our situations, both components of the binary are located inside the inner edge of the CB disk (Günther \& Kley 2002; Shi et al. 2012; Shi \& Krolik 2015). Therefore, we assume that there is a wide gap between the binary systems and the disk, and we neglect any mass inflow from the CB disk into the inner region (but also see the further discussion in Section 3). Because of Keplerian rotation, $\mathrm{CB}$ disks have a lower angular velocity than the binary systems. By the tidal interaction, the angular momentum is continuously transferred from the binary to the inner edge of the disk. Subsequently, the angular momentum is transported from the inner edge to the outer region of the disk by the waves and internal viscous stresses (Artymowicz \& Lubow 1994). As a result, the disk rapidly spreads outward to redistribute the angular momentum. The rate by which the orbital separation decays due to the resonant interaction between the binary and the CB disk is given by Lubow \& Artymowicz (1996) and Dermine et al. (2013) as

$$
\frac{\dot{a}}{a}=-\frac{2 l}{m} \frac{M_{\mathrm{d}} \alpha}{\mu}\left(\frac{H}{R}\right)^{2} \frac{a}{R} \Omega
$$

where $l$ and $m$ are the time-harmonic number and the azimuthal number (Artymowicz \& Lubow 1994), respectively; $M_{\mathrm{d}}$ and $\mu$ are the CB disk mass and the reduced mass of the binary, respectively; $\alpha$ is the viscous parameter, $\Omega$ is the orbital angular velocity, and $H$ and $R$ are the thickness and the half-angularmomentum radius of the disk, respectively. 
For a detached binary, Kepler's third law $\left(G\left(M_{1}+M_{2}\right) /\right.$ $a^{3}=4 \pi^{2} / P^{2}$ ) leads to

$$
\frac{\dot{P}}{P}=\frac{3}{2} \frac{\dot{a}}{a}
$$

The objects we explore in this study are all detached binaries with short orbital periods. We therefore assume that their eccentricities are small due to the tidal interactions. When the eccentricity $e \leqslant 0.1 \sqrt{\alpha}$, the resonances are very weak to drive the eccentricity, and $m=l$ (Dermine et al. 2013). ${ }^{4}$ Combining Equations (1) and (2) the predicted orbital-period derivative can be written as

$$
\dot{P}=-6 \pi \frac{M_{\mathrm{d}} \alpha}{R}\left(\frac{H}{R}\right)^{2} \frac{a}{\mu} .
$$

Equation (5) shows that the orbital-period derivative is determined by two factors: a degenerate factor that depends on a combination of the CB disk parameters $\left(\left(M_{\mathrm{d}} \alpha H^{2}\right) / R^{3}\right)$ and a parameter that depends on the properties of the binary $(a / \mu)$. Based on the measured CB disk mass in NN Ser, we can constrain the first factor and hence the CB disk parameters (i.e., $\alpha, H$, and $R$ ), and then use this calibration for the other six sources.

If the gas-to-dust ratio of a $\mathrm{CB}$ disk is similar to that of the interstellar medium ( 100, Muno \& Mauerhan 2006), the total mass of the CB disk in NN Ser can be estimated to be $2.4 \times 10^{-4} M_{\odot}$. Assuming that the orbital decay of NN Ser arises from the tidal torque of a CB disk, we obtain $\alpha H^{2} / R^{3}=1.9 \times 10^{-21} \mathrm{~cm}^{-1}$ from Equation (5). In this work, we adopt a relatively thin $\mathrm{CB}$ disk and low viscous parameter, i.e., $H / R=0.03, \alpha=0.01$, which yields $R=315$ au. The inner radius $\left(r_{\text {in }}\right)$ of the CB disk relies on the resonant torque between the binary and the $\mathrm{CB}$ disk, which pushes the disk outward. Based on SPH simulations for the inner radius of the CB disk at various eccentricities (Artymowicz \& Lubow 1994), Dermine et al. (2013) fitted a simple formula:

$$
r_{\text {in }}(e, \Re)=1.7+0.375 \log (\Re \sqrt{e}) \mathrm{au},
$$

where $\mathfrak{R}=(H / R)^{-2} \alpha^{-1} \approx 10^{5}$ is the Reynolds number of the CB disk gas. This implies that the inner radius of the CB disk is not sensitive to the eccentricity $\left(e=10^{-4}\right.$ leads to $r_{\text {in }}=2.825 \mathrm{au}$, whereas $e=10^{-6}$ yields $r_{\text {in }}=2.45 \mathrm{au}$ ). In this work, we take $e=10^{-4}$ and the outer radius of the disk $r_{\text {out }}=1000 \mathrm{au}, \quad$ so that $R=\left(r_{\text {in }}+r_{\text {out }}\right) / 4+\sqrt{r_{\text {in }} r_{\text {out }}} / 2 \approx$ $300 \mathrm{au},{ }^{5}$ which is in good agreement with the value of $R$ derived for NN Ser.

To examine whether a CB disk could be responsible for the orbital decay of our detached binaries, we show in Figure 1 a comparison of the predicted orbital-period derivatives in the CB disk model with observations in a $\dot{P}-a / \mu$ diagram. All theoretical curves (using Equation (3)) are based on the same CB disk parameter $\alpha H^{2} / R^{3}$ as deduced for NN Ser. The estimated CB disk mass in NN Ser $\left(M_{\mathrm{d}}=2.4 \times 10^{-4} M_{\odot}\right)$ is shown as a solid line. Since one would generally expect a range of CB disk masses for different systems, we also show $\dot{P}$ as a function of $a / \mu$ for disk masses varying by two orders of

\footnotetext{
4 For larger eccentricities $0.1 \sqrt{\alpha} \lesssim e \lesssim 0.2$, the $m=2, l=1$ resonance is the strongest contribution driving the eccentricity.

5 We assume that the relation between the surface density and the distance from the disk center obeys $\sigma(r) \propto r^{-2}$.
}

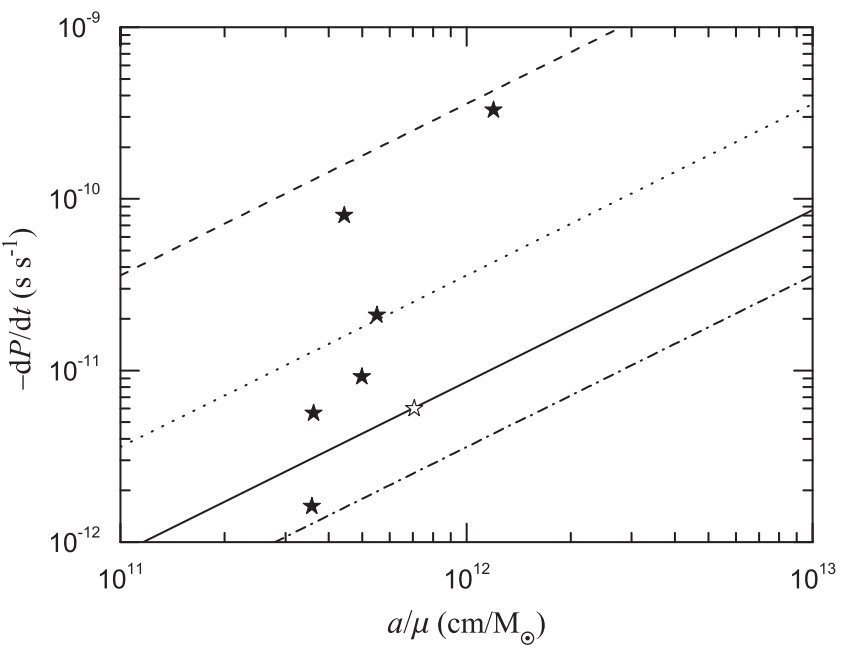

Figure 1. Comparison of the predicted orbital-period derivatives in the $\mathrm{CB}$ disk scenario with observations in the $\dot{P}-a / \mu$ diagram. The solid stars represent six detached binaries, the open star denotes NN Ser. The solid, dashed, dotted, and dashed-dotted curves represent $\mathrm{CB}$ disk masses of $2.4 \times 10^{-4}$ (the inferred $\mathrm{CB}$ disk mass for NN Ser), $10^{-2}, 10^{-3}$, and $10^{-4} M_{\odot}$, respectively.

magnitude: $10^{-2}$ (dashed line), $10^{-3}$ (dotted line), and $10^{-4} M_{\odot}$ (dashed-dotted line). Five of the detached binaries are located within a fairly narrow range of $\mathrm{CB}$ disk masses of $10^{-4}-10^{-3} M_{\odot}$, while both SDSS J143547.87+373338.5 and WY Cancri would require a rather heavy $\mathrm{CB}$ disk around $10^{-2} M_{\odot}$. This suggests that the CB disk model can in principle explain the observations for a range of assumed disk masses and be responsible for the observed orbital-period derivatives.

The dynamical interaction between the binary and the CB disk can induce an eccentricity in a binary system (Artymowicz \& Lubow 1994). However, the tidal force in a secondary star with a relatively deep convective envelope will try to circularize the orbit. The circularization timescale for a binary containing a $0.5 M_{\odot}$ main-sequence star and a $0.8 M_{\odot}$ white dwarf can be estimated as (Hurley et al. 2002)

$$
\tau_{\text {cir }} \approx 60\left(\frac{a}{R_{\odot}}\right)^{8} \text { year. }
$$

For a small eccentricity, $\dot{e}=-50 e \dot{a} /(\alpha a)$ (Lubow \& Artymowicz 1996), the timescale for driving eccentricity can be written as

$$
\tau_{\mathrm{ecc}} \approx \frac{e}{\dot{e}}=-\frac{\alpha a}{50 \dot{a}} .
$$

With the exception of WY Cancri, all other sources have a short circularization timescale, $\tau_{\text {cir }} \lesssim 40$ year. According to Equation (8), the eccentricity growth timescale is inversely proportional to $\dot{P}$. SDSS J143547.87+373338.5 has the shortest eccentricity growth timescale of $\tau_{\text {ecc }} \approx 1.3 \times$ $10^{4}$ year. Since $\tau_{\text {cir }} \ll \tau_{\text {ecc }}$, the tidal force of the secondary is sufficiently strong to keep the orbit approximately circular.

\section{Discussion and Summary}

In a previous study, we investigated whether a CB disk could be responsible for the large orbital-period derivative detected in NN Ser (Chen 2009) and WY Cancri (Chen 2013). We assumed that a fraction of the wind-loss from the donor star feeds the CB disk, which would yield a tidal torque by the 
interaction between the disk and the binary to extract orbital angular momentum. To produce the observed orbital-period derivatives, the donor stars would have to have a very high wind mass-loss rate and a relatively high fraction of the wind feeding the CB disk. Therefore, the possibility that the CB disk was fed by a wind alone to produce the rapid orbital decay observed in these two sources could be ruled out (Chen 2009, 2013).

In this Letter, we employed a very different CB disk model. Six of the seven detached binaries listed in Table 1 have ultracompact orbits (all sources except for WY Cancri have an orbital period less than 0.2 days), which are all likely to be post-common-envelope binaries. In the final stage of a common-envelope phase, a fraction of the orbital energy of the binary is believed to expel most of the envelope, giving rise to the birth of a compact binary (Ivanova et al. 2013). However, not all the CE material needs to be ejected, and the remaining material may collapse into a disk surrounding the binary, i.e., form a CB disk (Spruit \& Taam 2001) around the newly formed more compact, detached binary. Our estimates show that the existence of a CB disk can produce the observed orbital-period derivatives in such systems. The interaction torque between the binary and the CB disk plays a vital role in the shrinking of the binary orbit, similar to the conclusion given by Kashi \& Soker (2011). Based on the disk parameters derived by the observed orbital-period derivative and the inferred mass $\left(\sim 2.4 \times 10^{-4} M_{\odot}\right)$ of the candidate $\mathrm{CB}$ disk in NN Ser, the disk mass in seven sources could be constrained to be $10^{-4}-10^{-2} M_{\odot}$, which is close to the observed disk mass range $\left(10^{-4}-10^{-3} M_{\odot}\right.$; Gielen et al. 2007). The viscous timescale at the inner edge of the disk, $\tau=$ $\alpha^{-1}(H / R)^{-2}\left(r_{\text {in }} / a\right)^{3 / 2} \Omega^{-1}$, can be estimated to be $\sim 10^{5}$ year for NN Ser (adopting the same parameters as in Section 2). Haefner et al. (2004) obtained a cooling time of 1.3 Myr for the white dwarf in NN Ser. This implies that viscous torques operate sufficiently fast that they can successfully transport the angular momentum to the outer region of the disk, but also that the phase of fast orbital decay (at least several viscous timescales) is long enough to be observable in a system like NN Ser.

Our scenario neglected any gas flow in or across the gap between the disk and the binary. On the other hand, detailed simulations indicate that some gas at the inner boundary of the disk may penetrate the gap and be accreted onto one or both binary components (Günther \& Kley 2002; Shi et al. 2012; Shi \& Krolik 2015). Assuming the inflowing gas possesses the specific angular momentum $\left(j=\sqrt{G\left(M_{1}+M_{2}\right) r_{\text {in }}}\right)$ at the inner edge of the CB disk, the rate of orbital-period increase of the binary for an accretion rate $\dot{M}$ can be estimated to be

$$
\frac{\dot{P}}{P} \approx 3 \frac{\dot{J}_{\mathrm{acc}}}{J}=\frac{3 j \dot{M}}{\mu \sqrt{G\left(M_{1}+M_{2}\right) a}}=\frac{3 \dot{M}}{\mu}\left(\frac{r_{\mathrm{in}}}{a}\right)^{1 / 2},
$$

where $\dot{J}_{\text {acc }}$ is the angular-momentum-increasing rate from the accretion, and $J$ is the total angular momentum of the binary. For NN Ser, the gas inflow from the disk would not change the tendency of orbital decay if the accretion rate $\dot{M}<2.5 \times 10^{-11} M_{\odot} \mathrm{yr}^{-1}$. This threshold accretion rate is seven orders of magnitude higher than the inferred value $\left(\sim 9.0 \times 10^{-18} M_{\odot} \mathrm{yr}^{-1}\right)$ for a $\mathrm{CB}$ disk surrounding a white dwarf binary (Farihi et al. 2017). Therefore, it seems that the gas inflow of the CB disk cannot significantly alter the evolution of the binary in our scenario.

One might expect that the mass contained in a $\mathrm{CB}$ disk would evolve because of mass-loss via a disk wind. However, it is difficult to estimate the wind-loss rate at the moment. In principle, the change of the orbital-period derivative could be used to obtain an indirect estimate of the disk wind-loss rate. According to Equation (5), the $\mathrm{CB}$ disk mass changes according to $\Delta M_{\mathrm{d}} \propto \Delta \dot{P}$, where $\Delta \dot{P}$ is the change of the orbital-period derivative. V2051 Oph has the smallest estimated CB disk mass $\left(\sim 10^{-4} M_{\odot}\right)$ and therefore provides the best candidate for testing the CB disk scenario. If $\dot{P}$ decreases by a factor of $1 \%$ over a period of 1000 years, the CB disk mass would accordingly decrease by $1 \%$, producing a disk mass-loss rate of $\sim 10^{-9} M_{\odot} \mathrm{yr}^{-1}$.

In principle, our $\mathrm{CB}$ scenario strongly depends on the migration rate. Kocsis et al. (2012) simulated the co-evolution of binaries and CB disks and found that gas would pile up outside the binary's orbit rather than create a cavity for some system with smaller separations and masses. In this case, the disk properties are intermediate between the weakly perturbed case (Type I migration) and the case with a gap (Type II migration). As a result, the migration rate of the secondary in this "Type 1.5" regime is slower than both Type I and Type II rates. Therefore, the $\mathrm{CB}$ disk would not be responsible for the rapid orbital decay of these detached binaries if the migration rates were similar to a Type 1.5 rate. In addition, we neglect the persistent co-rotation torques, which should tend to drive an outward migration.

Besides the Applegate mechanism, a third body can also produce cyclic period changes in close binaries. For example, Qian et al. (2009) proposed that a tertiary component with a mass of $11.1 M_{\text {Jupiter }}$ orbiting NN Ser at an orbital radius of $d_{3}=3.29$ au could give rise to the observed orbital-period derivative. However, this variation would be periodic, not secular, and it should therefore be possible to distinguish this from genuine angular-momentum-loss from the binary. Hence, the observed orbital decays may provide indirect evidence for the existence of $\mathrm{CB}$ disks in these systems. Long-term observations should allow further strengthening of this conclusion. In addition, CB disks may be observable with $L$ band (3-4 $\mu \mathrm{m})$ observations, due to the continuum contribution of dust emission (Spruit \& Taam 2001). Therefore, future detailed multi-waveband observations for these detached binaries are required to confirm or refute our scenario.

We are grateful to the anonymous referee for the helpful comments that improved the manuscript. We also thank S.B. Qian for providing us with the relevant binary parameters for some detached binaries. This work was partly supported by the National Natural Science Foundation of China (under grant number 11573016), the Program for Innovative Research Team (in Science and Technology) at the University of Henan Province, and the China Scholarship Council. This work has also been supported by a Humboldt Research Award to Ph.P. at the University of Bonn.

\section{References}

Andronov, N., Pinsonneault, M., \& Sills, A. 2003, ApJ, 582, 358

Antoniadis, J. 2014, ApJL, 797, L24

Applegate, J. H. 1992, ApJ, 385, 621

Artymowicz, P., \& Lubow, S. H. 1994, ApJ, 421, 651 
Baptista, R., Santos, R. F., Fandez-Abans, M., \& Bortoletto, A. 2007, AJ, 134,867

Brinkworth, C. S., Marsh, T. R., Dhillon, V. S., \& Knigge, C. 2006, MNRAS, 365,287

Chen, W.-C. 2009, A\&A, 499, L1

Chen, W.-C. 2013, PASJ, 65, L4

Chen, W.-C., \& Li, X.-D. 2006, MNRAS, 373, 305

Chen, W.-C., \& Li, X.-D. 2007, ApJL, 658, L51

Chen, W.-C., \& Li, X.-D. 2015, A\&A, 583, A108

Chen, W.-C., Li, X.-D., \& Qian, S.-B. 2006, ApJ, 649, 973

Dermine, T., Izzard, R. G., Jorissen, A., \& van Winckel, H. 2013, A\&A, 551, A50

Eisenstein, D. J., Liebert, J., Harris, H. C., et al. 2006, ApJS, 167, 40

Farihi, J., Parsons, S. G., \& Gänsicke, B. T. 2017, NatAs, in press (arXiv:1612. 05259)

Gielen, C., van Winckel, H., Waters, L. B. F. M., Min, M., \& Dominik, C. 2007, A\&A, 475, 629

Günther, R., \& Kley, W. 2002, A\&A, 387, 550

Haefner, R. 1989, A\&A, 213, L15

Haefner, R., Fiedler, A., Butler, K., \& Barwig, H. 2004, A\&A, 428, 181

Hardy, A., Schreiber, M. R., Parsons, S. G., et al. 2016, MNRAS, 459, 4518

Heckert, P. A., Maloney, G. V., Stewart, M. C., et al. 1998, AJ, 115, 1145

Hurley, J. R., Tout, C. A., \& Pols, O. R. 2002, MNRAS, 329, 897

Ivanova, N., Justham, S., Chen, X., et al. 2013, A\&ARv, 21, 59

Kashi, A., \& Soker, N. 2011, MNRAS, 417, 1466

Kilkenny, D., O’Donoghue, D., Koen, C., Lynas-Gray, A. E., \& van Wyk, F. 1998, MNRAS, 296, 329

King, A. R., Schenker, K., \& Hameury, J. M. B. 2002, MNRAS, 335, 513
Kocsis, B., Haiman, Z., \& Loeb, A. 2012, MNRAS, 427, 2660

Lubow, S. H., \& Artymowicz, P. 1996, in NATO ASIC Proc. 477, Evolutionary Processes in Binary Stars, ed. R. A. M. J. Wijers, M. B. Davies, \& C. A. Tout (Dordrecht: Kluwer), 53

Muno, M. P., \& Mauerhan, J. 2006, ApJL, 648, L135

Pretorius, M. L., \& Knigge, C. 2008, MNRAS, 385, 1485

Pyrzas, S., Gäsicke, B. T., Marsh, T. R., et al. 2009, MNRAS, 394, 978

Qian, S.-B., Dai, Z.-B., Liao, W.-P., et al. 2009, ApJL, 706, L96

Qian, S.-B., Dai, Z.-B., Zhu, L.-Y., et al. 2008, ApJL, 689, L49

Qian, S.-B., Han, Z.-T., Fernández-Lajús, E., et al. 2015, ApJS, 221, 17

Qian, S.-B., Han, Z.-T., Soonthornthum, B., et al. 2016, ApJ, 817, 151

Qian, S.-B., Liu, L., Liao, W.-P., et al. 2011, MNRAS, 414, L16

Qian, S.-B., Zhu, L.-Y., Dai, Z.-B., et al. 2012, ApJL, 745, L23

Rappaport, S., Joss, P. C., \& Verbunt, F. 1983, ApJ, 275, 713

Schwarz, R., Schwope, A. D., Vogel, J., et al. 2009, A\&A, 496, 833

Shi, J.-M., \& Krolik, J. H. 2015, ApJ, 807, 131

Shi, J.-M., Krolik, J. H., Lubow, S. H., \& Hawley, J. F. 2012, ApJ, 749, 118

Spruit, H. C., \& Ritter, H. 1983, A\&A, 124, 267

Spruit, H. C., \& Taam, R. E. 2001, ApJ, 548, 900

Steinfadt, J. D. R., Bildsten, L., \& Howell, S. B. 2008, ApJL, 677, L113

Taam, R. E., \& Spruit, H. C. 2001, ApJ, 561, 329

van den Heuvel, E. P. J. 1994, in Interacting Binaries, ed. S. N. Shore et al. (Berlin: Springer), 263

van den Heuvel, E. P. J., \& de Loore, C. 1973, A\&A, 25, 387

Verbunt, F., \& Zwaan, C. 1981, A\&A, 100, L7

Wang, X., \& Wang, Z. 2014, ApJ, 788, 184

Wood, J. H., \& Saffer, R. 1999, MNRAS, 305, 820

Zeilik, M., Cox, D. A., Ledlow, M. J., et al. 1990, ApJ, 363, 647 